\title{
The quality of dairy products made from the milk of cows consuming vitamin-containing preparations
}

\author{
Lydia G. Kashirina*, Konstantin A. Ivanishchev, and Kirill I. Romanov \\ Ryazan State Agrotechnological University named after P.A. Kostychev, 390044 Ryazan, Russia
}

\begin{abstract}
The article contains information on the state of the processes of lipid peroxidation (LPO) in fresh cows under the influence of stress reaction caused by labor and the beginning of the lactation period. To reduce oxidative stress in the body of fresh cows, antioxidants were used in the form of preparations "E-selenium" and "Butofan". The aim of the research was to determine the effect of vitamin-containing preparations "E-selenium" and "Butofan" on the productivity of fresh cows, the quality of milk and butter, made from it. The studies were carried out during the winter-stall period on one of the farms of Ryazan district of Ryazan region and involved 12 fresh black-and-white cows aged 4 years. The control group of animals was intact. As a result of the investigations, it was found that due to the action of the above preparations, which have antioxidant properties, the productivity parameters in the experimental groups of animals were higher compared to the control group, because they had an inhibiting effect on lipid peroxidation processes in the organism and activated the work of its own antioxidant system.
\end{abstract}

\section{Introduction}

At present, the relationship between biochemical blood parameters in the body of cows and productivity is well studied. To improve the productivity and the quality of milk, various preparations are used and their effect on metabolic processes in the body of animals, in particular lactating cows, is studied $[1,2]$. The processes of lipid peroxidation (LPO) occur constantly in all living organisms, however, in our opinion, the process remains understudied. With the participation of free radicals, the process is carried out by a chain reaction mechanism, which proceeds in three stages: the first one is the start, the second one is the chain branching and the third one is the chain breaking. At the first stage, mainly diene conjugates (DC) are formed. These are primary and most important products of peroxidation. The formation of products of the secondary oxidation of lipids - malonic dialdehyde (MDA) - finishes the process. Strengthening LPO processes leads to a whole complex of changes at the cellular level: reduction of activity of a number of enzymes, damage and inhibition of DNA synthesis, lysis of cellular structures, disruption of cell division processes, inhibition of protein and fat synthesis in the body, which leads to some decrease in quality composition of milk [3].

In addition, the LPO products, DC in particular, have detergent properties, namely, they destroy the membrane of fat globules and contribute to their fragmentation, which ultimately reduces the fat content of milk, worsening its technological properties and dairy products yield [4]. LPO products have positive properties. They are necessary for implementation of many metabolic processes in the body of animals, but their formation in large quantities has a toxic effect [4].

To protect against excessive formation of free radicals in living organisms, an antioxidant system exists. With prolonged exposure of a living organism of adverse factors, its functional reserves may become depleted and the body will not fully cope with the regulation of free radical reactions. At the same time, oxidative stress may occur, which will lead to disruption of cell membrane structures, entailing the accumulation of toxic substances in cells, which are not being fully broken down by enzymes will linger in the body. It will affect the development of oxidative stress, and subsequently, can affect the health and productivity of animals [3,5]. A number of factors is known that contribute to the enhancement of LPO in animals, in particular, the effect of physical exertion, but the effect of age features and various physiological states of the body on these processes has not been discovered.

In a normally functioning organism, the LPO processes proceed at low speed in those cells where there are mainly phospholipids, i.e. unsaturated lipids. They affect the renewal of the cellular membranes of the body, their permeability, the regulation of the growth rate and processes of cell proliferation. Antioxidants reduce the concentration of free radicals and lipid peroxidation products and thereby affect the basal metabolism in favor of assimilation processes, which entails an increase in the productive qualities of dairy cattle [6]. 
In order to resist oxidative stress during periods of intense physiological load, it is necessary to introduce additional antioxidant preparations for animals [7]. A decrease in the antioxidant activity of the body can occur under conditions of spring deficit of antioxidants, stress, physical inactivity and excessive caloric intake. During such periods, there is an increase in free radical oxidation, a decrease in enzymatic oxidation, an increase in the utilization of oxygen in the body, which is explained by the disparity between the excess of fatty acids and oxygen in the tissue and the level of their consumption.

In our experimental studies, preparations Butofan and E-selenium were used. Butotan as an "antioxidant" was used due to its unusual composition, which includes butafosfan and vitamin $\mathrm{B}_{12}$ (cyanocobalamin). Butofosfan influences a number of metabolic processes: fat, protein and carbohydrate, stimulates them and increases the resistance of the organism. Vitamin $B_{12}$ is involved in the activation of blood formation processes and methionine synthesis, influences the formation of glycogen in DNA synthesis. Quite often, this veterinary preparation is used as a maintenance therapy for various pathologies.

The composition of preparation "E-selenium" includes vitamin $\mathrm{E}$ or tocopherol, which is a powerful antioxidant and prevents the spread of reactive oxygen species. Selenium that is a part of the preparation is also involved in the formation of the antioxidant system, affects the immune system, carbohydrate and fat metabolism, enhances the effect of a number of vitamins, and is included in many hormones.

\section{Aim and objectives}

The aim of the research was to determine the effect of preparations "E-selenium" and "Butofan" on the productivity of fresh cows and the quality of milk and butter made of it. Research objectives included:

- determining the content of catalase and $\alpha$ tocopherol in the blood plasma of cows under the influence of preparations;

- determining the effect of the above preparations on the yield of cows;

- identification of the effect of preparations on the fractional composition of lipid globules in milk;

- determining the fatty acid composition of the butter made of cows' milk under the influence of these preparations.

The choice of the above preparations was determined by their composition. "E-selenium" contains active antioxidants sodium selenite and tocopherol acetate. It affects carbohydrate and fat metabolism, regulates redox processes, enhances the effect of vitamin A and has some positive effect on the immune system. "Butofan" contains butafosfan and cyanocobalamin. Butafosfan stimulates metabolic processes in the body. Cyanocobalamin activates the formation of vitamin B12, blood formation processes, etc. The above preparations are manufactured by domestic manufacturers, but are not used as antioxidants.

\section{Materials and methods}

The experiment was carried out with 12 fresh black-andwhite cows - counterparts aged 3 years in LLC Zarya, Ryazan District, Ryazan Region. Animals were divided into three groups of 4 heads each - two experimental ones and the control.

The experiment lasted for 150 days. All animals were clinically healthy. Animal rations were nutritionally balanced and met the standards of the RAAS [8]. The cows of the control group were intact, experimental animals were injected with E-selenium intramuscularly once a month for 5 months at a dose of $10 \mathrm{ml}$ per head, starting from the second month of lactation. The second experimental group was administered Butophan at the same dosage, but twice a month, because it does not have cumulative properties and has a rapid half-life.

Blood sampling was carried out from the jugular vein in the morning before feeding at the end of each month of lactation after calving, for 5 months starting from the second one. Blood samples were stabilized with a $10 \%$ solution of ethylene diaminetetraacetic acid (EDTA). Blood plasma and milk were examined with a ChemWell biochemical analyzer and ApelPD-303 UV spectrophotometer. To determine the concentration of malonic dialdehyde (MDA), diene conjugates (DC) and $\alpha$-tocopherol in blood plasma and milk, the optical density of the solutions was determined with ApelPD303 UV spectrophotometer.

In order to study the work of the antioxidant system of the animal organisms, the content of catalase and ceruloplasmin was determined in the blood plasma with the help of ChemWell biochemical analyzer.

The content of fat and protein in milk samples was determined with "Laktan1-4" device.

The number and size of milk fat globules was determined using a Goryaev chamber. Milk diluted with distilled water 25 times, filled into the camera and photographed with a microscope with a $40 \times$ lens and a $15 \times$ eyepiece. The total number of milk fat globules was calculated on the obtained images, their diameter was determined and distributed into 10 classes depending on the diameter.

The data obtained in the course of the research were processed statistically using Student's methods (Rokitskiy, P.F., 1967). The difference between the three groups of cows, one control and two experimental ones, and between the periods of sampling was determined. The difference with $\mathrm{P}<0.05$ was considered reliable. The calculations were performed using STATISTICS and Microsoft Office Excel 2003 software packages.

Studies of blood plasma and milk were carried out in the interdepartmental research laboratory of nanotechnology at the Faculty of Veterinary Medicine and Biotechnology at Ryazan State Agrotechnological University.

At the end of the 1st, 3rd and 5th months of lactation after calving, milk samples in the amount of 101 were taken from which sweet-butter was made in the traditional way. Analysis of the fatty acid composition of the butter was carried out at Moscow Independent Laboratory of Raw Material and Food Products Quality 
with the help of a hardware-software complex for medical research based on Chromatek-Crystal 5000 chromatograph according to GOST 301663-2012; GOST 30418-96; GOST 30623-98; GOST R 51483-99.

\section{Results and discussion}

Vitamin E or $\alpha$-tocopherol is involved in the stabilization of cell membranes by slowing the oxidation of polyunsaturated fatty acids. Catalase is an enzyme that provides the decomposition of hydrogen peroxide into molecular oxygen and water. Hydrogen peroxide, having accumulated in the body, leads to destruction and cell death. Catalase prevents this process. Starting from the third month of lactation, the activity of catalase in the blood plasma of cows of the experimental groups under the influence of antioxidants was higher than in the control. This trend continued in the subsequent months.

Table 1. Catalase activity in plasma, $\mu \mathrm{mol} / \mathrm{l}(\mathrm{n}=4)$

\begin{tabular}{|c|c|c|c|c|}
\hline \multirow{2}{*}{ Group } & \multicolumn{4}{|c|}{ Lactation month after calving } \\
\cline { 2 - 5 } & 2 & 3 & 4 & 5 \\
\hline Control & $23.4 \pm 2.3$ & $22.5 \pm 3.9$ & $16.9 \pm 7.3$ & $16.2 \pm 3.3$ \\
\hline Experimental 1 & $24.4 \pm 2.7$ & $26.9 \pm 4.2$ & $27.0 \pm 9.3$ & $26.2 \pm 4.6$ \\
\hline Experimental 2 & $23.1 \pm 1.8$ & $27.6 \pm 4.0 *$ & $32.5 \pm 4.9$ & $33.9 \pm 3.8 * *$ \\
\hline
\end{tabular}

Note: The reliability of the difference of parameters compared with the control group $*-p \leq 0.05, * *-p \leq 0.01$.

During the whole experiment the content of $\alpha$ tocopherol in the blood plasma of cows of experimental group 2, administered with "Butofan", varied over a wider range, and was higher than that in other groups. In the 5th month, it was significantly higher compared with other groups (Table 2).

Table 2. The content of $\alpha$-tocopherol in the blood plasma of cows, $\mu \mathrm{mol} / 1(\mathrm{n}=4)$

\begin{tabular}{|c|c|c|c|c|}
\hline \multirow{2}{*}{ Group } & \multicolumn{4}{|c|}{ Lactation month after calving } \\
\cline { 2 - 5 } & 2 & 3 & 4 & 5 \\
\hline Control & $102.1 \pm 5.2$ & $82.6 \pm 3.0 * *$ & $80.5 \pm 2.9^{* *}$ & $81.5 \pm 3.2$ \\
\hline $\begin{array}{c}\text { Experimental } \\
1\end{array}$ & $101.7 \pm 3.4$ & $83.1 \pm 7.6$ & $89.6 \pm 3.7$ & $83.9 \pm 7.2$ \\
\hline $\begin{array}{c}\text { Experimental } \\
2\end{array}$ & $102.7 \pm 5.3$ & $83.5 \pm 11.2$ & $90.4 \pm 8.1$ & $84.3 \pm 6.9 * * *$ \\
\hline
\end{tabular}

Note: The reliability of the difference of parameters compared with the control group $* *-p \leq 0.01, * * *-p \leq 0.001$.

Vitamin E, which is included in preparation "Eselenium", was able to compensate for the production of other antioxidants and controlled the level of lipid peroxidation in the blood plasma of cows during the entire experiment. As well as "Butofan", which maintained a high level of the main antioxidants in the blood plasma of cows of experimental group 2 .

Since milk is a blood product, the effect of antioxidants on the productivity of fresh cows was determined. It has been established that "the height of lactation" fell on the third month of lactation after calving, i.e., in February. In the following months, there was a period of decline in productivity in all groups, but under the influence of vitamin-containing preparations, the experimental groups had a higher level (Fig. 1).

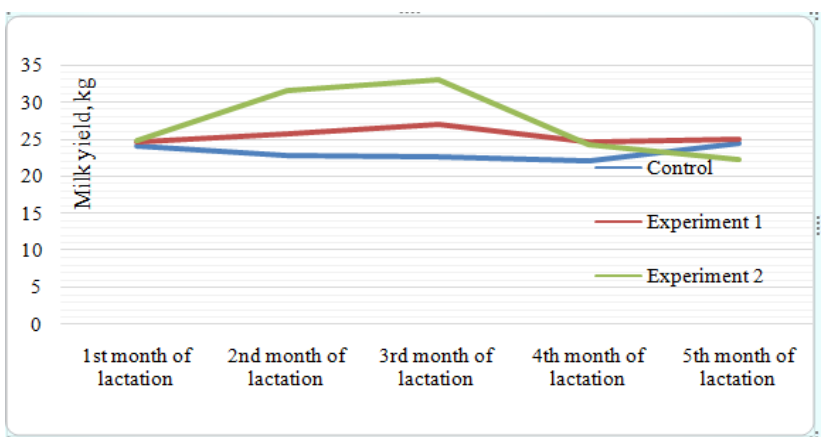

Fig. 1. Dynamics of daily milk yield by months of lactation

Milk is an emulsion of fat globules in the milk plasma. Plasma is a milk fluid that is free of fat, and all other parts of the milk are unchanged. The average diameter of the fat globules is from 2.0 to 2.5 microns. Their size and quantity in milk are unstable and depend on the physiological characteristics of animals and zootechnical factors, which are of practical importance in the transition of fat into milk products. In the process of free radical oxidation, milk fat undergoes autooxidation, which subsequently leads to milk spoilage and the resulting dairy products. Antioxidants provide delayed oxidative deterioration of the product. Milk contains a number of natural antioxidants - these are tocopherols, ascorbic acid, carotene, phospholipids, and others, but in most cases they are not enough to inhibit oxidative processes.

As a part of our experiment, studies of the dispersion of milk fat in cow's milk under the influence of antioxidants were carried out (Table 2).

The dispersion of fat globules depends on the breed of animals, lactation stage, conditions of livestock, etc. [5]. Beside the standard requirements for butter production, there are some special ones: the fat content, the degree of dispersion of fat globules and the composition of milk fat.

Under the influence of antioxidants, an increase in the number of fat globules was observed in the milk of cows of the experimental groups, obtained in the third month of lactation, as compared with the control. This trend remained in next months.

From the 3rd month of lactation, the control group of intact cows had higher intensity of lipid peroxidation (LPO) processes compared with these parameters in animals of the experimental groups, which resulted in a decrease in size and number of fat globules.

Due to the action of the above preparations with antioxidant properties, the productivity in the experimental groups was higher compared with the control in the subsequent months of lactation, because they had an inhibitory effect on the processes of lipid peroxidation in the body of animals, activating the work of their own antioxidant system at different stages and free radicals did not have time to accumulate in large quantities. 
Table 3. Dispersion of milk fat

\begin{tabular}{|c|c|c|c|c|c|}
\hline Group of cows & \begin{tabular}{|c|} 
The number of milk \\
fat globules, billion/ml
\end{tabular} & $\begin{array}{c}\text { The average diameter } \\
\text { of the fat globule, } \\
\text { microns }\end{array}$ & $\begin{array}{l}\text { The share of small } \\
\text { globules (up to } \\
2 \text { microns), } \%\end{array}$ & $\begin{array}{c}\text { The share of medium } \\
\text { globules } \\
(2-3 \text { microns }), \%\end{array}$ & $\begin{array}{c}\text { The share of large } \\
\text { globules } \\
\text { (> } 3 \text { microns), } \%\end{array}$ \\
\hline \multicolumn{6}{|c|}{$1^{\text {st }}$ month of lactation } \\
\hline Control & $2.669 \pm 158$ & $4.24 \pm 0.06$ & 8.16 & 15.46 & 76.8 \\
\hline Experiment 1 & $2.631 \pm 132$ & $4.24 \pm 0.08$ & 8.19 & 14.98 & 76.6 \\
\hline Experiment 2 & $2.639 \pm 139$ & $4.25 \pm 0.05$ & 8.16 & 15.45 & 77.1 \\
\hline \multicolumn{6}{|c|}{$2^{\text {nd }}$ month of lactation } \\
\hline Control & $2.641 \pm 0.141$ & $5.31 \pm 0.06$ & 3.46 & 9.92 & 86.62 \\
\hline Experiment 1 & $2.657 \pm 0.147$ & $5.27 \pm 0.07$ & 3.52 & 9.87 & 85.89 \\
\hline Experiment 2 & $2.612 \pm 0.131$ & $5.33 \pm 0.04$ & 3.47 & 9.92 & $76.22 *$ \\
\hline \multicolumn{6}{|c|}{$3^{\text {rd }}$ month of lactation } \\
\hline Control & $2.630 \pm 0.133$ & $5.12 \pm 0.06$ & 3.94 & 9.53 & 86.52 \\
\hline Experiment 1 & $2.677 \pm 0.153$ & $5.30 \pm 0.08$ & 3.29 & 9.80 & 89.64 \\
\hline Experiment 2 & $3.058 \pm 0.157^{* * *}$ & $5.28 \pm 0.04$ & 3.35 & 9.78 & 90.23 \\
\hline \multicolumn{6}{|c|}{$4^{\text {th }}$ month of lactation } \\
\hline Control & $2.598 \pm 0.134$ & $5.4 \pm 0.06$ & 4.72 & 12.3 & 82.94 \\
\hline Experiment 1 & $2.671 \pm 0.160$ & $5.19 \pm 0.06$ & 4.12 & 14.7 & 88.51 \\
\hline Experiment 2 & $2.675 \pm 0.162$ & $5.21 \pm 0.07$ & 3.98 & 15.12 & 88.81 \\
\hline \multicolumn{6}{|c|}{$5^{\text {th }}$ month of lactation } \\
\hline Control & $2.638 \pm 0.138$ & $5.15 \pm 0.04$ & 4.51 & 11.8 & 82.95 \\
\hline Experiment 1 & $2.896 \pm 0.159$ & $5.25 \pm 0.08$ & 4.15 & 14.4 & 86.21 \\
\hline Experiment 2 & $2.642 \pm 0.143$ & $5.19 \pm 0.06$ & 4.07 & 15.2 & 85.31 \\
\hline
\end{tabular}

Note: The reliability of the difference of parameters compared with the control group $*-p \leq 0.05, * *-p \leq 0.01$.

To determine the effect of antioxidants on the quality of dairy products, butter was made in which the composition of polyunsaturated fatty acids (PUFAs) was determined. Unsaturated fatty acids have an effect on improving immunity and lowering cholesterol levels in the blood. Linoleic, linolenic and arachidonic acids are the main representatives of PUFA, they are part of cellular structures, are involved in metabolism and provide growth processes. PUFAs are not synthesized by the human body, and therefore are considered indispensable along with some amino acids and vitamins.

Arachidonic acid has the greatest biological activity, which is scarce in food, but with the participation of vitamin $\mathrm{B}_{6}$ it can be synthesized by the body from linoleic acid. The concentration of linoleic acid in butter was the highest in all groups.

The composition of milk fat influences the taste of butter, its consistency and storage stability [8]. Milk fat is a mixture of glycerides, free fatty acids, stearins and other substances. About 150 saturated and unsaturated fatty acids are found in milk fat.

A distinctive feature of polyunsaturated acids is that they are not produced by the human body and must come from the outside with food. Saturated (marginal) fatty acids are fatty acids having no double bonds in the structure [6]. The uncontrolled use of saturated fatty acids can cause development of many diseases. But one should not completely remove them from the diet, because they are involved in synthesis of hormones, transport and assimilation of vitamins and microelements, and are also a source of energy [7].

The butter made of the milk of cows of different groups had an increase in the concentration of saturated fatty acids by $5.7 \%$ by the end of the studies in the control group. There was a decrease in this parameter in milk from cows of experimental group 1 by $6.5 \%$ and in experimental group 2 by $7.9 \%$.

Saturated and unsaturated fatty acids in the body of animals are interrelated and some of them can be transformed into others [9]. The experimental groups had an increase in the concentration of unsaturated fatty acids compared with the control: experimental group 1 by $16.6 \%$ and experimental group 2 by $31.1 \%$.

We believe that these changes occurred under the influence of antioxidant preparations "E-Selen" and "Butofan", which reduced LPO reactions and thus protected unsaturated acids from oxidation, increasing their content in butter. The results are improved quality and taste parameters of butter, which are also important.

Table 4. Changes in the concentration of fatty acids in butter, $\%$

\begin{tabular}{|c|c|c|c|}
\hline \multirow{2}{*}{} & \multicolumn{3}{|c|}{ Total PUFAs } \\
\cline { 2 - 4 } & Control & Experiment 1 & Experiment 2 \\
\hline January & $58.36 \pm 0.42$ & $54.64 \pm 0.51$ & $64.22 \pm 0.47$ \\
\hline March & $63.52 \pm 0.37$ & $62.47 \pm 0.40^{* *}$ & $64.25 \pm 0.42^{*}$ \\
\hline May & $61.38 \pm 0.4$ & $58.2 \pm 0.37$ & $59.11 \pm 0.39$ \\
\hline & \multicolumn{3}{|c|}{ Total NonPUFAs } \\
\hline January & $26.19 \pm 0.44$ & $25.31 \pm 0.47$ & $23.6 \pm 0.52$ \\
\hline March & $27 \pm 0.56^{*}$ & $28.13 \pm 0.61^{* *}$ & $27.13 \pm 0.58$ \\
\hline May & $28.92 \pm 0.65$ & $29.53 \pm 0.58$ & $30.95 \pm 0.61^{* *}$ \\
\hline
\end{tabular}

Note: The reliability of the difference of parameters compared with the control group $*-p \leq 0.05, * *-p \leq 0.01$.

\section{Conclusion}

To increase the productivity of cows and production of milk with improved biological qualities, manufacturers 
use various methods and preparations, which often have a negative impact on animal health. The use of antioxidants is one of the ways to increase the productivity and preserve the health of animals.

In our studies, there was a decrease in productivity at the 3rd month of lactation in the control group of intact animals by $2.22 \%$, in experimental group 2 milk yield decreased by $8.5 \%$ and in experimental group 3 there was a decrease in productivity by $26.6 \%$. However, compared to the control, in the 4th month there was an increase in milk yield in experimental group 2 by $12.2 \%$ and in experimental group 3 by $10 \%$.

Antioxidant preparations "E-selenium" and "Butofan" normalized metabolic and regenerative processes in the body, had a stimulating effect on metabolic processes, reducing the intensity of lipid peroxidation in plasma of lactating cows. Experimental studies have established that use of E-selenium and Butofan preparations had a positive effect on the reduction of lipid peroxidation processes in fresh cows. Butofan was the most active preparation. Its use continued to maintain antioxidant performance in the body of fresh cows at a high level until the end of the experiment. So, in the 3 months of lactation the activity of catalase in the blood plasma of cows of the experimental groups under the influence of antioxidants was higher than in the control by $22.66 \%$ in Experiment 2 and by $19.55 \%$ in Experiment 1 continuing to maintain this trend in the next months.

The content of $\alpha$-tocopherol in the blood plasma of cows of experimental group 2, which were administered Butofan, throughout the experiment varied within a wider range and was higher than in other groups. In the 5th month it was significantly higher than the control by $3.43 \%$ and by $0.47 \%$ compared to the group where Eselenium was used. There is also an increase in this parameter in experiment 1 by $2.94 \%$ compared with the control in the 5th month. An increase in the number of fat globules with some predominance of a large fraction was observed in the milk of cows in the experimental groups, especially in the experimental group 2. The difference with the control in the 3rd month of lactation was $16.27 \%$. This caused the increase in the amount of unsaturated fatty acids in butter, which improved its quality parameters. The changes in the fatty acid composition of butter, made from the milk of cows of different groups, were found.

The content of saturated fatty acids in butter made of milk from cows of the experimental groups was lower compared to the control by $6.5 \%$ in experimental group 1 and by $7.9 \%$ in experimental group 2. A $5.7 \%$ increase in the concentration of saturated fatty acids was observed in the control group of animals. An increase in the concentration of unsaturated fatty acids in the experimental group 1 was by $16.6 \%$ and the in experimental group 2 by $31.1 \%$, which is a positive factor, since these acids are most important for the organism of animals and humans.

Antioxidants are most appropriate to introduce during calving, because that is the time when oxidative reactions occur most intensively in cows' bodies. Paracmastic processes in the mammary gland are accompanied by an increase in lipid peroxidation products [10]. Antioxidant preparations E-selenium and Butofan, tested in experimental studies, normalized the metabolic and regenerative processes in the body, had a stimulating effect on metabolism, contributing to an increase in the amount of unsaturated fatty acids in milk and products derived from it. Consequently, the use of antioxidants in the practice of dairy cattle breeding contributes to the preservation of animal health and the production of high quality products.

\section{References}

1. N.N. Alekseeva, Changes in the activity of ceruloplasmin in the blood serum under the influence of various factors Hygiene and Sanitation 8, 70-71 (1991)

2. R.Kh. Karmoliev, Biochemical processes during free radical oxidation and antioxidant protection. Prevention of oxidative stress in animals Agricult. Biology 2, 19-28 (2002)

3. L.G. Kashirina, A.V. Antonov, I.A. Pluschik, Effect of lipid peroxidation on the quality of milk fat in cows, "Innovative directions and methods for the implementation of scientific research in the agroindustrial complex" in Coll. of sci. works of RSATU teachers and graduate students 378-381 (2012)

4. L.G. Kashirina, K.A. Ivanishchev, The effect of antioxidants in the form of vitamin-containing preparations on quality parameters of milk and the fatty acid composition of cottage cheese made from it Herald of RSATU named after P.A. Kostychev 2, 142-148 (2018)

5. P.F. Rokitskiy, Biological statistics (High School, Minsk, 1967)

6. A.Kh. Babaeva, Lipid peroxidation and antioxidant protection in the blood serum when toxemia of pregnancy and iron deficiency anemia Bull. of probl. of biology and med. 23-26 (2017)

7. I.A. Zbarovskiy, M.V. Bannikov, The antioxidant system of the body, its role in metabolism Monthly Sci. J. 3, 18-22 (2014)

8. G. Maranon, W. Manley, P. Cayado et al., Equine colic induces intestinal apoptosis via a mitochondria-mediated pathway in Proc. of 10th Int. Congr. of World Equine Vet. Ass. (Moscow, 2008)

9. V.S. Ulitko, S.P. Lifanova, The effect of using antioxidant carotene-containing preparations on milk productivity of cows Herald of Ulyanovsk State Agricult. Acad. 2-5 (2014)

10. B. Kucsunska, T. Nalecs-Taruacka, Relationship between content of phospholipids in milk and stability of milk fat globule membrane and the effects of some factors on the frequency of their destabilization Annals of Warshaw agr. univ. 289-294 (Warshaw, 2009) 